\title{
Receptor-Mediated Endocytosis of Two-Dimensional Nanomaterials Undergoes Flat Vesiculation and Occurs by Revolution and Self-Rotation
}

\author{
Jian Mao, ${ }^{\dagger}$ Pengyu Chen,${ }^{\dagger}$ Junshi Liang, Ruohai Guo and Li-Tang Yan* \\ Key Laboratory of Advanced Materials (MOE), Department of Chemical Engineering, \\ Tsinghua University, Beijing 100084, P. R. China
}

The files include:

1. Supplementary Discussions and Derivation

SI-1: Models of coarse-grained graphene nanosheet, lipid and receptor (Page 3)

SI-2: Details of DPD simulations (Page 5)

SI-3: GN-bilayer interaction energy analysis during GN revolution (Page 7)

SI-4: GN-bilayer interaction energy analysis during the GN piercing process (Page 12)

2. Supplementary Videos S1-S4

3. References 


\section{SI-1. Models of coarse-grained graphene nanosheet, lipid and receptor}

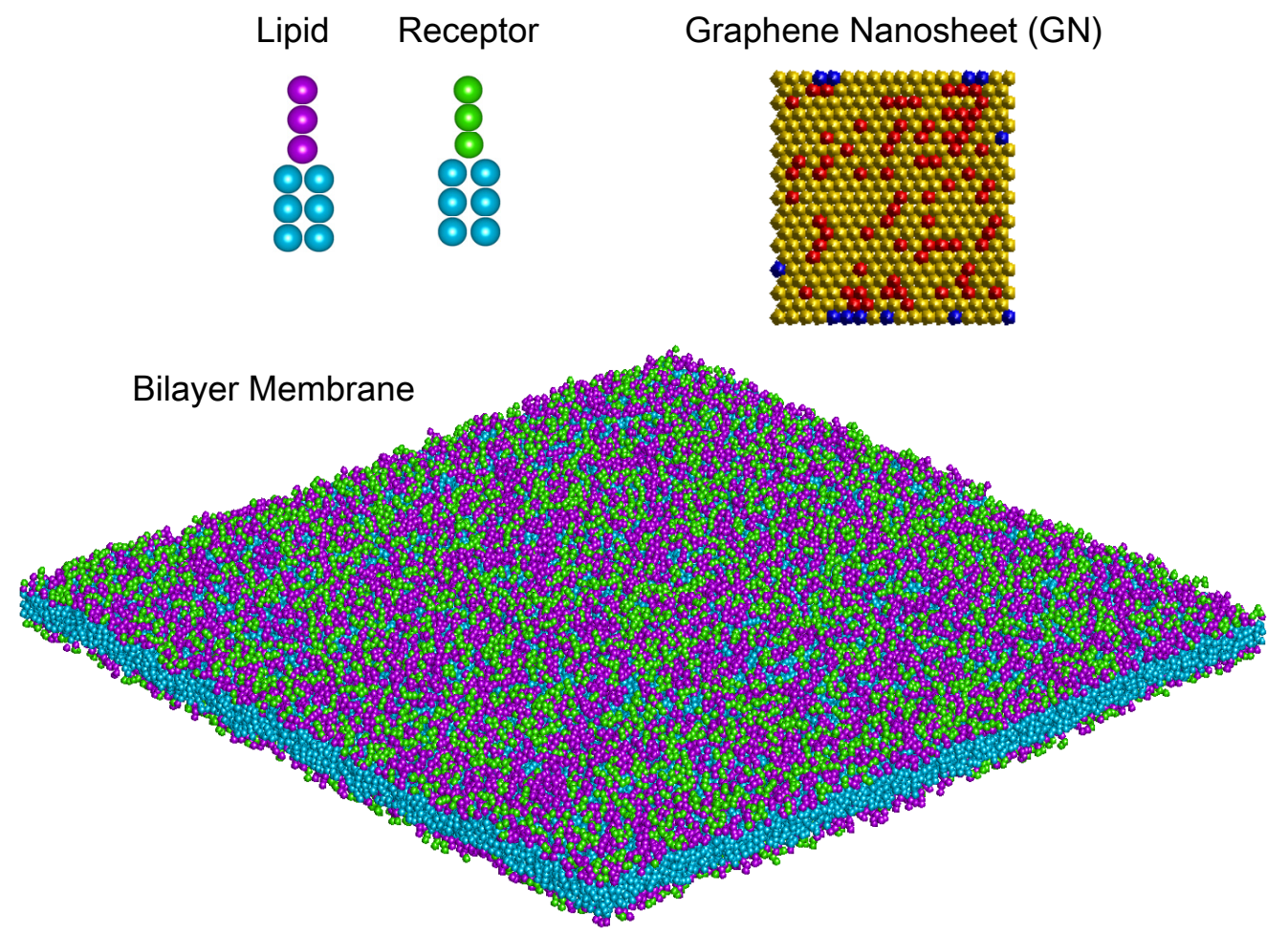

Figure S1. Coarse-grained (CG) molecular models for the representative entities used in the simulations. The model of amphiphilic lipid is constructed by a head group with three hydrophilic beads (pink) and two tails consisting of three hydrophobic beads (cyan). The receptors are modeled as the same as lipids except having a different head group (green). Each graphene nanosheet (GN) is modeled by arranging the beads on a single layer of FCC lattice into a desired area. The colors of yellow, blue, and red indicate the beads of the unoxidized basal plane, the oxidized edge, and the oxidized basal plane of GN. 10,000 lipids and receptors with density $f_{R}$ self-assemble into a lipid bilayer membrane spanning the simulation box.

In the present paper, our coarse-grained simulations are on the basis of dissipative particle dynamics (DPD), ${ }^{1}$ which is a Lagrangian method derived from coarse-graining of molecular dynamics widely used as a mesoscopic simulation method for biomembrane systems. In our DPD model, each amphiphilic lipid consists of a head group and two tails as 
shown in Fig.S1. ${ }^{2-4}$ The head group is constructed by three connected hydrophilic beads and each tail includes three connected hydrophobic beads. The receptors are modeled as the same as the lipids except having a different GN-receptor attractive head group. ${ }^{2}$ 10,000 lipids and receptors with various densities self-assemble into a lipid bilayer membrane spanning the simulation box. To mimic a real cell membrane with a larger area-to-volume ratio, $N$-varied DPD method is used in our simulations, where the targeted membrane tension is maintained by monitoring the lipid number per area.,

As an example of 2D nanomaterials, a sparsely oxidized GN is used in the present simulations, which is constructed based on the typical structure model representing outcomes from standard oxidization processes. ${ }^{7,8,9}$ Moreover the Young's modulus of the GN model has been calibrated according to the experimentally found elasticity of graphene. ${ }^{10-12}$ Experiments on bulk graphite give about $1 \mathrm{TPa}$ for the in-plane Young's modulus, ${ }^{13}$ while the Young's modulus of graphene varies between 0.5 and $1.0 \mathrm{TPa}^{14,15}$ In order to have a similar Young's modulus of our GN model, the modulus of the spring and the modulus of the angle bend between beads of the GN are selected as $700 \mathrm{kcal} \cdot \mathrm{mol}^{-1} \cdot \AA^{-2}$ and $700 \mathrm{kcal} \cdot \mathrm{mol}^{-1} \cdot \mathrm{rad}^{-2}$ respectively. ${ }^{10}$ 


\section{SI-2. Details of DPD simulations}

In a DPD system, a set of interacting beads is considered, whose time evolution is governed by the following Newton's motion equations: ${ }^{1}$

$$
\frac{d \mathbf{r}_{i}}{d t}=\mathbf{v}_{i}, \frac{d \mathbf{v}_{i}}{d t}=\mathbf{f}_{i}
$$

Furthermore the total force $f_{l}$ acting on every bead can be expressed by ${ }^{12}$

$$
f_{i}=\sum_{j \neq i}\left(F_{i j}^{C}+F_{i j}^{D}+F_{i j}^{R}\right)+F_{i}^{S}+F_{i}^{A}
$$

where $F_{i j}^{C}, F_{i j}^{D}, F_{i j}^{R}, F_{i}^{S}, F_{i}^{A}$ are the conservative force, the dissipative force, the random force, the spring force and the angle force respectively. Here $F_{i}^{S}, F_{i}^{A}$ are additional forces, which are introduced to represent the interactions between bonded beads. The conservative force $F_{i j}^{C}$, which is derived from a soft interaction potential within the certain cutoff radius $r_{c}$, can be given by the following equation:

$$
F_{i j}^{C}=\left\{\begin{array}{l}
a_{i j}\left(1-\frac{r_{i j}}{r_{c}}\right) e_{i j} \quad\left(r_{i j}<r_{c}\right) \\
0\left(r_{i j} \geq r_{c}\right)
\end{array}\right.
$$

where $\mathbf{r}_{i j}=\mathbf{r}_{i}-\mathbf{r}_{j}, \quad r_{i j}=\left|\mathbf{r}_{i j}\right|$ and $e_{i j}=\mathbf{r}_{i j} / r_{i j} . a_{i j}$ is the maximum repulsion between bead $i$ and bead $j$, which is related with the Flory-Huggins $\chi$-parameter $\left(\chi_{i j}\right)$ as follow

$$
\chi_{i j}=\frac{\alpha_{i j}-\alpha_{i i}}{3.27}
$$

where $a_{i i}$ is the repulsion parameter between the same type beads and $a_{i i}=25$ is used in our DPD system. $\alpha_{i j}$ will be smaller than 25 for a strong attraction between two beads while it will be larger than 25 for a strong bead-bead repulsion interaction. Furthermore, the decrease of $\alpha_{i j}$ corresponds to the enhancement of bead attraction and vice versus. In our CG simulations, each parameter is selected carefully based on the properties and interactions of different beads. ${ }^{10,11}$ In particular, the parameters for the interactions of lipid 
head-unoxidized graphene, lipid tail-unoxidized graphene, lipid head-oxidized graphene, and lipid tail-oxidized graphene are $28,21,25$, and 26, respectively. In order to represent the strong binding affinity between receptors and GN beads, the GN-receptor interaction parameter has been set to five., ${ }^{2,6}$

The dissipative force $F_{i j}^{D}$ and the random force $F_{i j}^{C}$ act together as a thermostat and can be given by the following formulas:

$$
\begin{aligned}
& F_{i j}^{D}=-1 / 2 \sigma^{2} \omega^{D}\left(r_{i j}\right)\left(v_{i j} \cdot e_{i j}\right) e_{i j} \\
& F_{i j}^{R}=\sigma \omega^{R}\left(r_{i j}\right) \xi_{i j} \Delta t^{-1 / 2} e_{i j}
\end{aligned}
$$

where $v_{i j}=v_{i}-v_{j}$ and $\xi_{i j}$ is a random number which has zero mean and unit variance. The noise amplitude $\sigma$ is fixed at 3 and we take

$$
w^{D}\left(r_{i j}\right)=\left[w^{R}\left(r_{i j}\right)\right]^{2}=\left\{\begin{array}{l}
\left(1-\frac{r_{i j}}{r_{c}}\right)^{2} \quad\left(r_{i j}<r_{c}\right) \\
0\left(r_{i j} \geq r_{c}\right)
\end{array}\right.
$$

This particular thermostat is special in that is conserved angular momentum, which leads to a correct description of hydrodynamics.

In our simulations, a modified velocity-Verlet algorithm due to Groot and Warren is used to solve the motion equation. ${ }^{1}$ In details, we take the bead mass, the cutoff radius and the temperature as units and define the time scale as $\tau=\sqrt{m r_{c}{ }^{2} / k_{B} T}$. The size of our simulation box is $80 \times 80 \times 40 r_{c}^{3}$ and periodic boundary condition in all directions is taken into account. To prevent the passage of fluid beads through the GN, an additional bounce-back boundary condition is imposed on the GN surfaces. ${ }^{16}$ In order to assure the accurate temperature control of the simulation system, we set $\Delta t=0.02 \tau$ as the time step $^{17}$ and select a bead number density of $3 / r^{3}$. 
The reduced DPD units can be converted to SI units by examining the membrane thickness and the lipid diffusion coefficient. In a tensionless dipalmitoylphosphatidylcholine (DPPC) membrane, the typical area per lipid is about $0.64 \mathrm{~nm} .{ }^{18}$ We use this value to estimate the physical length of our simulations and get that $r_{c}$ is about $0.7 \mathrm{~nm}$. Thereby the area of the planar membrane in our simulation is about $56 \mathrm{~nm} \times 56 \mathrm{~nm}$. Moreover, we relate the time unit $\tau$ to the physical time by comparing the in-plane diffusion coefficient of lipids. A typical experimental result of this diffusion coefficient is $5 \mu \mathrm{m}^{2} / \mathrm{s} .{ }^{19}$ Thus we can get $\tau=7.7 \mathrm{~ns}$ and the total physical time of each calculation is over $60 \mu s\left(4 \times 10^{5}\right.$ time steps $)$.

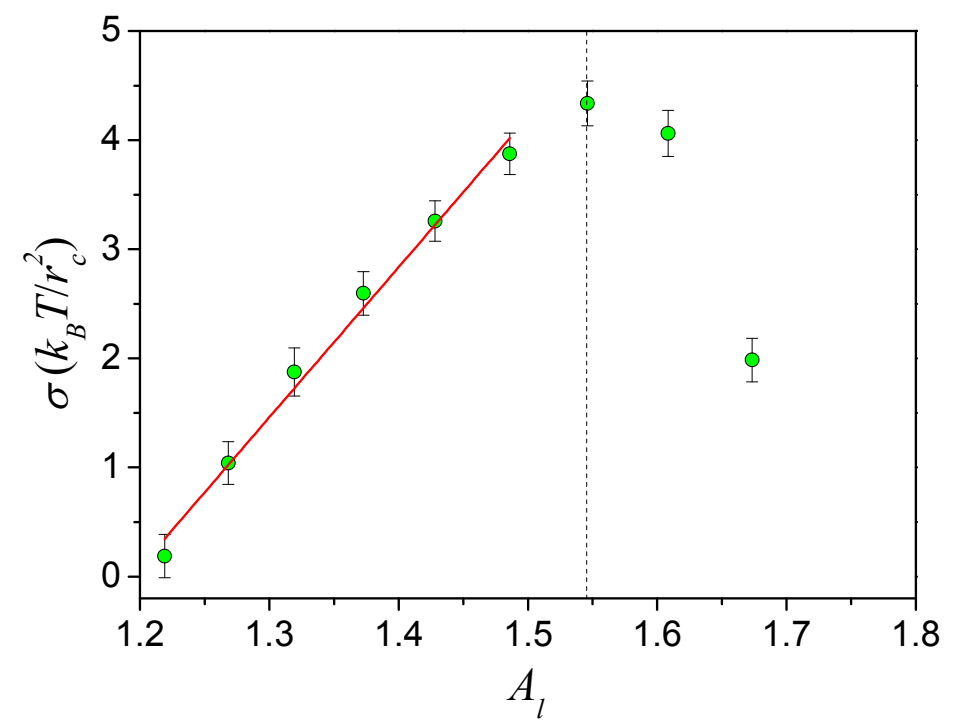

Figure S2. Membrane tension, $\sigma$, as a function of the area per lipid, $A_{l}$. The red line represents a linear fit for the points before the membrane rupture at the high surface tension as marked by the dashed line. 


\section{SI-3. GN-bilayer interaction energy analysis during GN revolution}
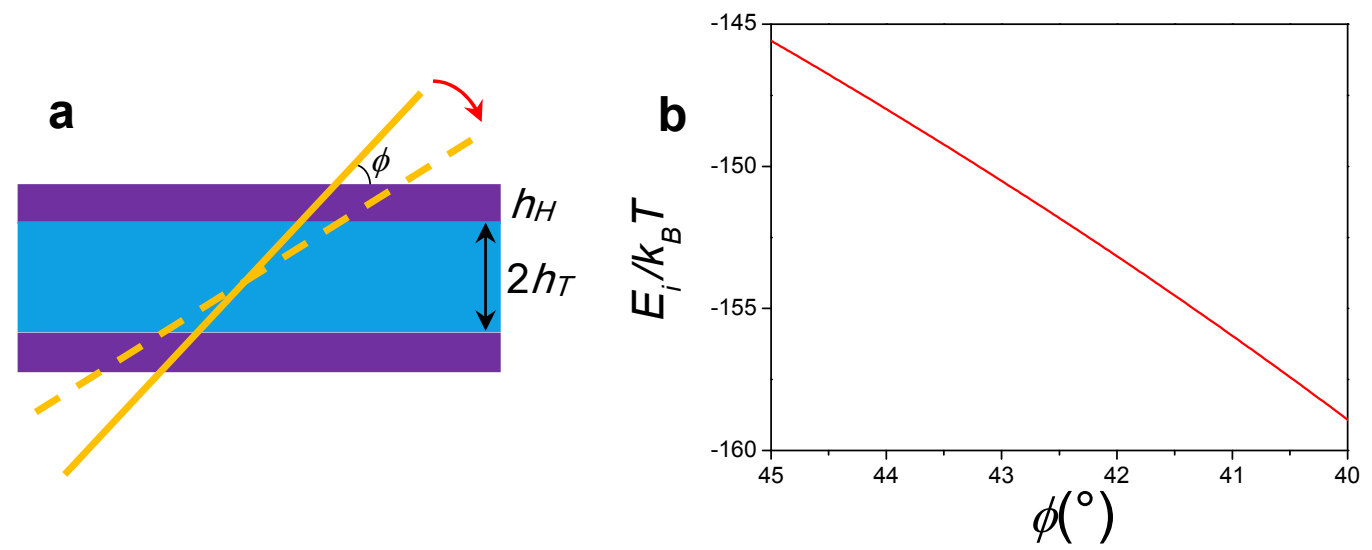

Figure S3. GN-bilayer interaction energy analysis during GN revolution relative to the membrane plane. a, The theoretical model denotes the rotation of the GN. $\phi$ represents the GN entry angle. The red arrow indicates the direction of GN rotation. b, The interaction energy, $E_{i}$, as a function of $\phi$ during GN revolution relative to the membrane plane.

The simulation result in Fig. $5 \mathrm{a}_{4}$ shows that a rotation of GN will occur after the GN diagonal parallel to the membrane surface reaches the middle of the membrane. Here we develop a theoretical model to analyze the GN-bilayer interaction energy change during the rotation of GN as shown in Fig.S3a. Because the rotation of GN leads to a change of the contact area between the GN and the bilayer, the GN-bilayer interaction energy change can be thereby obtained through multiplying by the interaction energy density. The interaction energy $E_{i}$ is related to terms of the following parameters: $l_{g}, \phi, \varphi$ (the edge length of GN, the entry angle of GN relative to the membrane plane, and the oxidized degree of GN), $h_{H}, h_{T}$ (the thicknesses of the head and the tail groups in the lipid monolayer respectively), $k_{H}, k_{T}$ (the interaction energy densities of the unoxidized basal plane of GN with the head groups and with the tail groups of the lipids respectively), $k_{H o}, k_{T o}$ (the interaction energy densities of the oxidized part of GN with the two groups of the lipids). Here we 
take $k_{H}=-k_{T}, k_{T o}=-k_{T}, k_{H o}=-k_{T o}=k_{T}\left(k_{T}<0\right)$ and $h_{T}=3 h_{H}$. Thus the GN-bilayer interaction energy $E_{i}$ during the rotating process of GN can be described by the following equation:

$$
E_{i}=8 k_{T}(1-2 \varphi) \frac{1}{\sin ^{2} \phi}\left[\sqrt{2} l_{g} \sin \phi h_{H}-h_{H}^{2}\right]
$$

Note that the oxidized degree $\varphi=0.5$ is a critical value. When $\varphi<0.5$, the GN-bilayer interaction energy will be negative, indicating that the hydrophobic attraction between lipid tails and the plane of GN dominates the GN-bilayer interaction process and drives the GN to pierce into the membrane spontaneously. However, when $\varphi>0.5$, the GN-bilayer interaction energy will be positive. In this case, the piercing will not happen and the GN turns to adhere to membrane surface. Thereby, $\varphi<0.5$ is assumed as the necessary condition in the energy analysis. In our simulations, we take the oxidized degree 0.3 and thereby the GN can pierce into the membrane spontaneously.

For the present simulations, the edge length is $l_{g}=7 \mathrm{~nm}$ and the oxidized degree is $\varphi=0.3$. We take typical parameter values as $k_{T}=-7 k_{B} T / \mathrm{nm}^{2}, h_{H}=0.5 \mathrm{~nm}^{20}$ As shown in Fig. $5 \mathrm{a}_{4}-\mathrm{a}_{5}$, the GN rotates from $45^{\circ}$ to $40^{\circ}$ relative to the membrane. Based on Eq. (8), we can get the plot of GN-bilayer interaction energy $E_{i}$ as a function of entry angle $\phi$ during the rotation of GN (Fig. S3b). It can be easily found that rotating GN from $45^{\circ}$ to $40^{\circ}$ leads to a significant decrease of the GN-bilayer interaction energy with about $13 k_{B} T$, which, fundamentally, can be attributed to the strong hydrophobic attraction of lipid tails with the GN. 


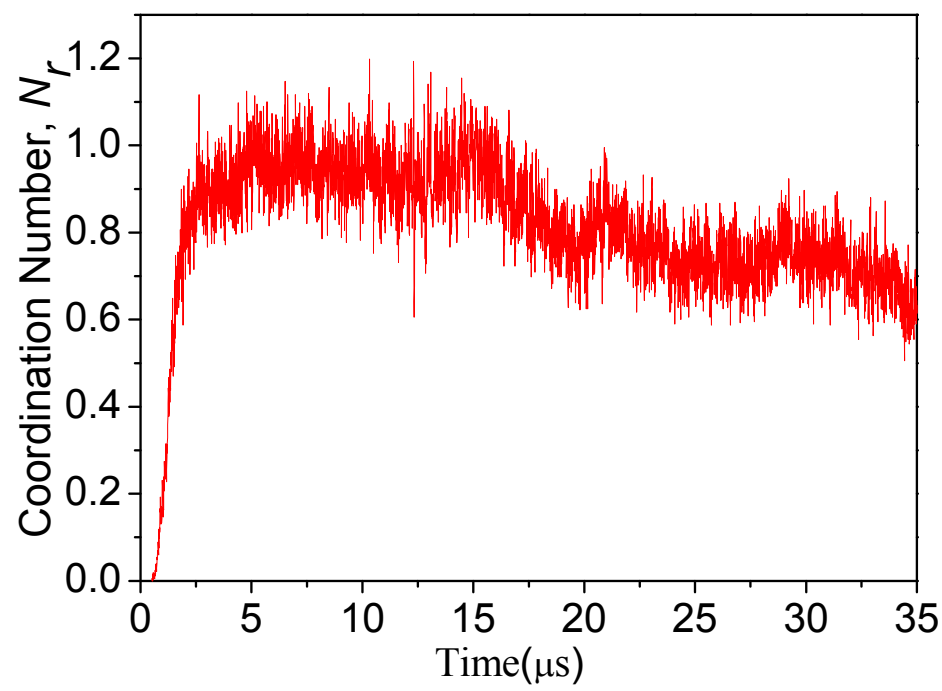

Figure S4. Receptor diffusion towards the basal plane of GN during flat vesiculation. In the event, GN with lateral size $l_{g}=7 \mathrm{~nm}$ is fully wrapped by a tense membrane with surface tension $\sigma=-0.65 k_{B} T / r_{c}^{2}$ and receptor density $f_{R}=0.5$. The plot shows the time evolution of the coordination number, $N_{r}$, of the GN during the vesiculation event. 


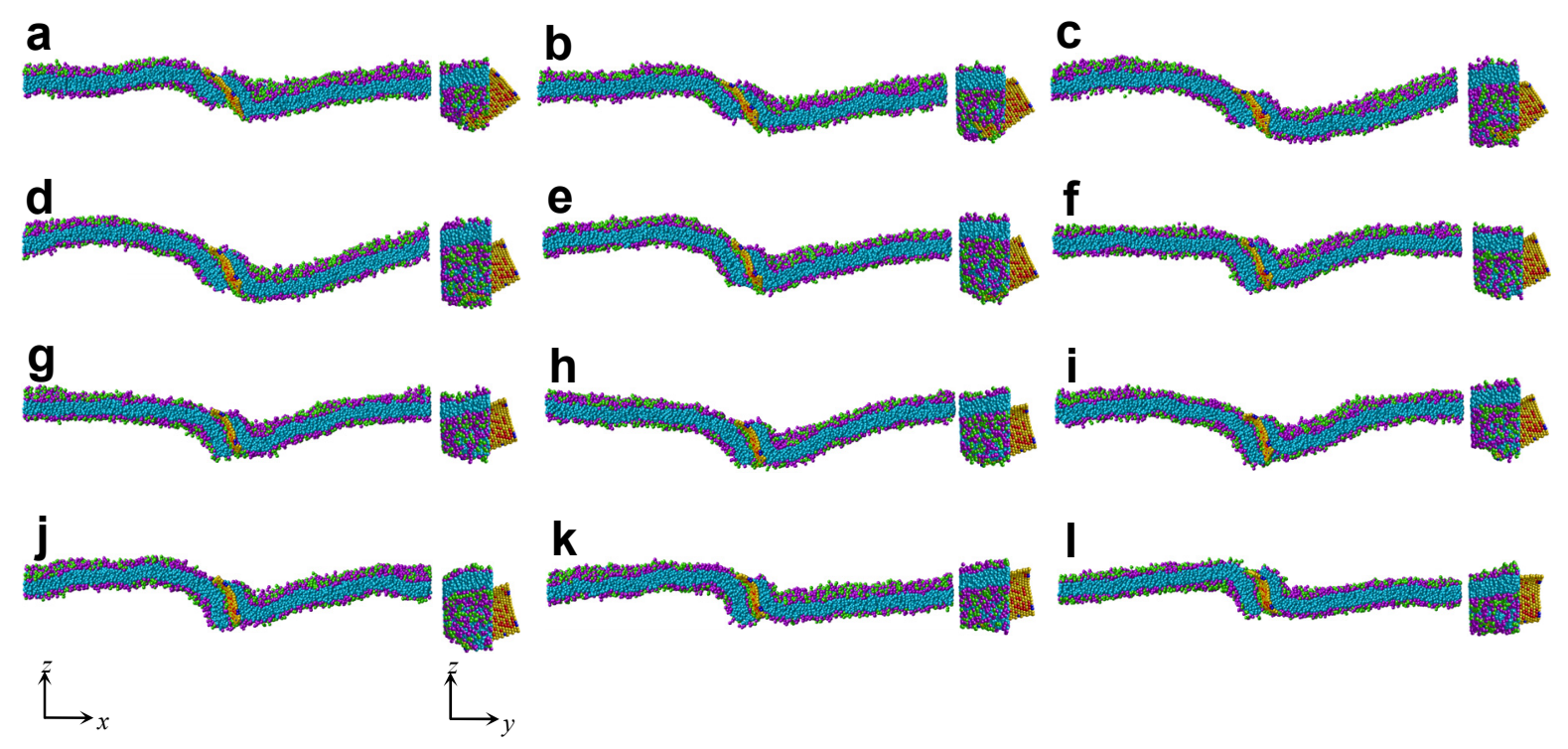

Figure S5. Revolution and self-rotation of GN during a vesiculation event towards incomplete flat vesicle. In the event, GN with lateral size $l_{g}=7 \mathrm{~nm}$ is partially wrapped by a tensionless membrane with receptor density $f_{R}=0.5$. The $x-z$ and $y-z$ cross section views of the lipid membrane around the GN are shown at each time for clarity, showing the revolution of the GN relative to the membrane and its self-rotation around the normal direction across its center, respectively. The times are: a, $23.10 \mu \mathrm{s} ; \mathbf{b}, 24.65 \mu \mathrm{s} ; \mathbf{c}, 26.18 \mu \mathrm{s} ; \mathbf{d}, 27.73 \mu \mathrm{s} ; \mathbf{e}, 30.80 \mu \mathrm{s}$; f, $33.88 \mu \mathrm{s} ; \mathbf{g}, 36.95 \mu \mathrm{s} ; \mathbf{h}, 40.05 \mu \mathrm{s} ; \mathbf{i}, 43.13 \mu \mathrm{s} ; \mathbf{j}, 46.20 \mu \mathrm{s} ; \mathbf{k}, 49.28 \mu \mathrm{s}$; and $\mathbf{l}, 53.90 \mu \mathrm{s}$. The color code of beads is the same as that of Fig.1. Solvent beads are not shown for clarity. 


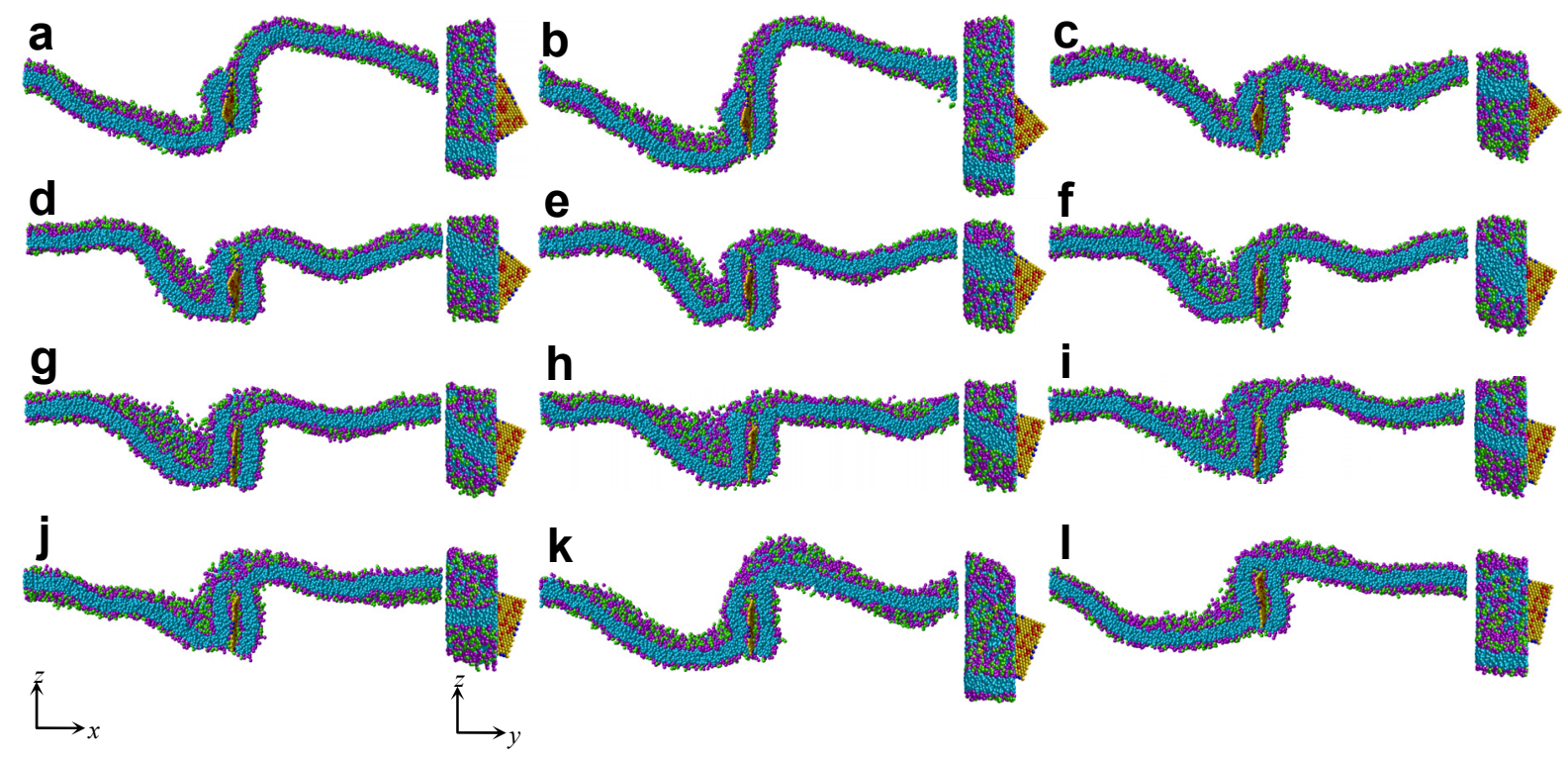

Figure S6. Revolution and self-rotation of GN during a vesiculation event towards flat vesicle. In the event, GN with lateral size $l_{g}=7 \mathrm{~nm}$ is fully wrapped by a tense membrane with surface tension $\sigma=-0.65 k_{B} T / r_{c}^{2}$ and receptor density $f_{R}=0.5$. The $x-z$ and $y-z$ cross section views of the lipid membrane around the GN are shown at each time for clarity, showing the revolution of the GN relative to the membrane and its self-rotation around the normal direction across its center, respectively. The times are: $\mathbf{a}, 26.18 \mu \mathrm{s} ; \mathbf{b}, 30.80 \mu \mathrm{s} ; \mathbf{c}, 30.85 \mu \mathrm{s} ; \mathbf{d}$, $40.05 \mu \mathrm{s} ; \mathbf{e}, 41.58 \mu \mathrm{s} ; \mathbf{f}, 43.13 \mu \mathrm{s} ; \mathbf{g}, 44.65 \mu \mathrm{s} ; \mathbf{h}, 46.20 \mu \mathrm{s} ; \mathbf{i}, 47.75 \mu \mathrm{s} ; \mathbf{f}, 49.28 \mu \mathrm{s} ; \mathbf{g}, 56.98 \mu \mathrm{s} ;$ and $\mathbf{h}, 61.60 \mu \mathrm{s}$. The color code of beads is the same as that of Fig.1. Solvent beads are not shown for clarity. 


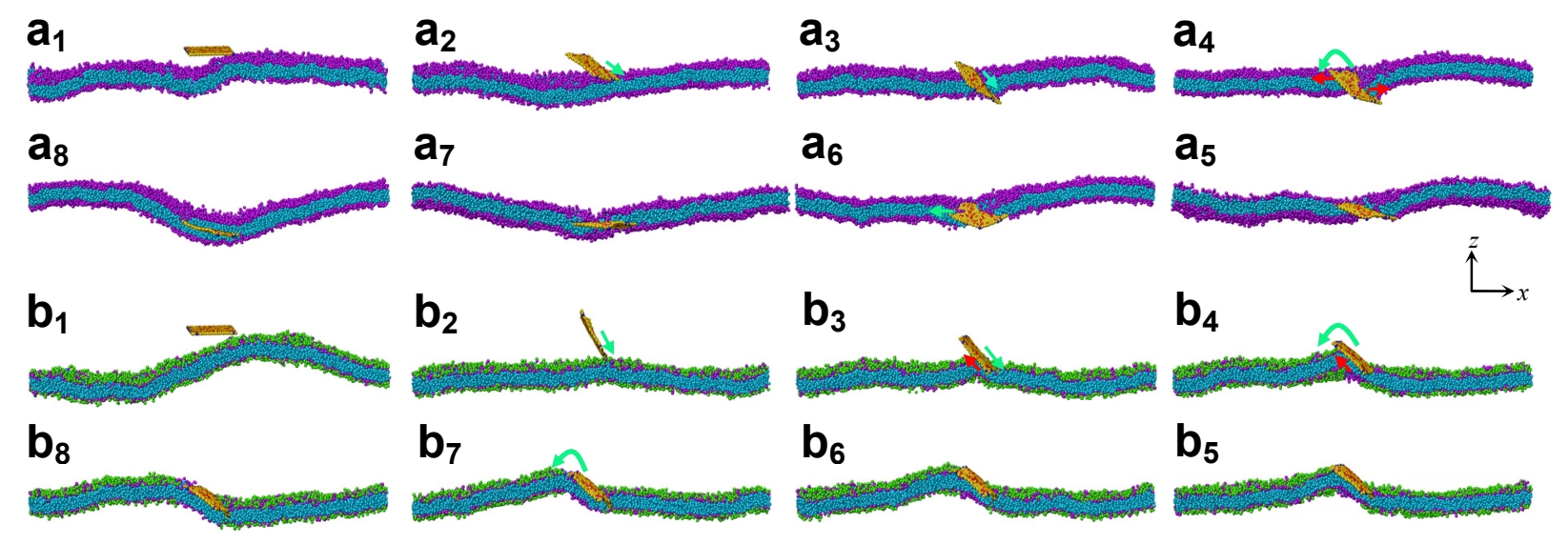

Figure S7. $\mathbf{a}_{1}-\mathbf{a}_{8}$, Successive stages of a vesiculation event where GN with lateral size $l_{g}=7 \mathrm{~nm}$ inserts into the center of a bilayer membrane without receptor density. The red and green arrows indicate the motion directions of the membrane and the GN respectively. The times of the slices are: $\mathbf{a}_{1}, 0 \mu \mathrm{s} ; \mathbf{a}_{2}, 2.30 \mu \mathrm{s} ; \mathbf{a}_{3}, 4.63 \mu \mathrm{s} ; \mathbf{a}_{4}, 5.78 \mu \mathrm{s} ; \mathbf{a}_{5}, 7.25 \mu \mathrm{s} ; \mathbf{a}_{6}, 19.25 ; \mathbf{a}_{7}$, $23.10 \mu \mathrm{s}$; and $\mathbf{a}_{8}, 28.65 \mu \mathrm{s}$. $\mathbf{b}_{\mathbf{1}}-\mathbf{b}_{\mathbf{8}}$, Successive stages in a interacting event where GN with lateral size $l_{g}=7 \mathrm{~nm}$ interacts with a bilayer membrane of $f_{R}=0.9$. The times are: $\mathbf{b}_{1}, 0 \mu \mathrm{s} ; \mathbf{b}_{\mathbf{2}}$, $0.25 \mu \mathrm{s} ; \mathbf{b}_{3}, 1.5 \mu \mathrm{s} ; \mathbf{b}_{4}, 15.10 \mu \mathrm{s} ; \mathbf{b}_{5}, 26.18 \mu \mathrm{s} ; \mathbf{b}_{6}, 28.50 \mu \mathrm{s} ; \mathbf{b}_{7}, 30.20 \mu \mathrm{s} ;$ and $\mathbf{b}_{\mathbf{8}}, 41.60 \mu \mathrm{s}$. Only the $x-z$ cross section view of the lipid membrane around the GN is shown in each snapshot for clarity. 


\section{SI-4. GN-bilayer interaction energy analysis during the GN piercing process}

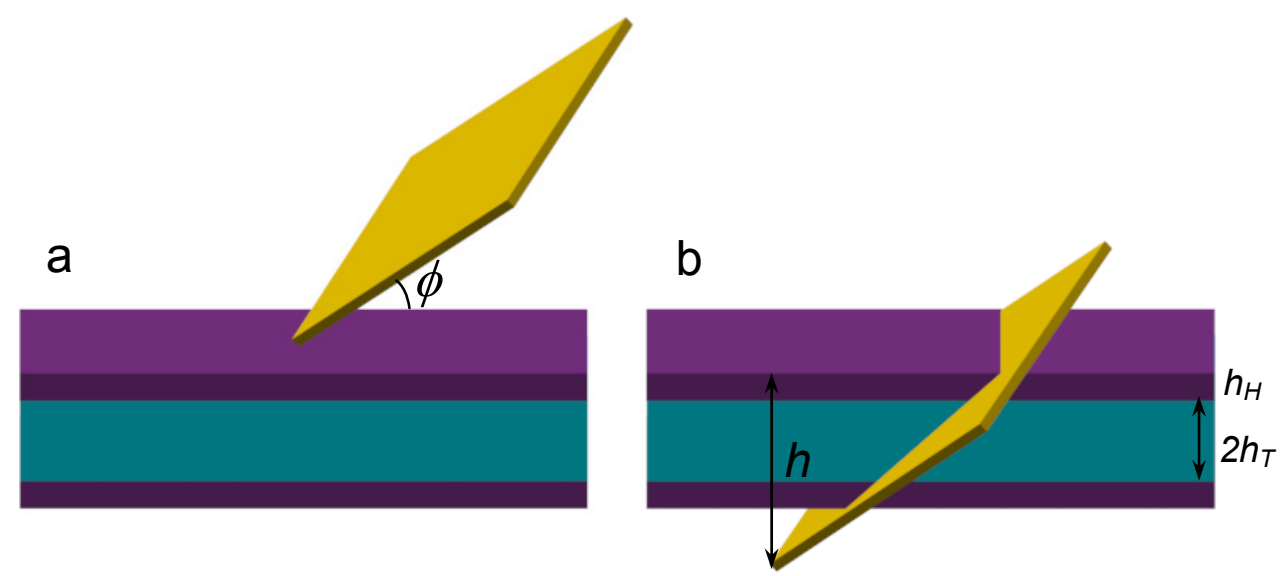

Figure S8. Theoretical model of GN-bilayer interaction energy analysis during the GN piercing process. a, The initial state of the GN piercing process where one of GN's corner sites just contacts the membrane. b, The final state of the piercing process where the GN's diagonal parallel to the membrane surface reaches the middle of the membrane. $h_{H}$ and $h_{T}$ are the thicknesses of the head and the tail groups in the lipid monolayer respectively. $h$ is the piercing depth from the membrane surface to the GN tip, and $\phi$ represents the GN entry angle.

In this section, a theoretical model is constructed to analyze the interrelated effects of GN sizes and the piercing depth on the GN-bilayer interaction energy during the piercing process. The GN starts piercing into the membrane through one of its corner sites with a predominant entry angle $\phi$ and keeps the pose during the piercing process as shown in Fig.S8a. The Fig.S8b shows the final state of the whole piercing process where the GN's diagonal parallel to the membrane surface reaches the middle of the membrane. Here we define $L_{d}=\sqrt{2} l_{g}$ as the diagonal length of the square GN and $L$ as the effective length of $L_{d}$ in the vertical direction against the membrane surface. Obviously, $L=L_{d} \sin \phi=\sqrt{2} l_{g} \sin \phi$. In our energy analysis, we study the GN size from $L>2 h_{T}$. The GN-bilayer interaction energy 
can be calculated by integrating the energy density along the contacting area between GN and different parts of the membrane as demonstrated in Fig.S8b. The following piecewise functions can thereby be obtained to describe the interaction energy, $E_{i}^{\prime}$, versus GN size and the piercing depth during the piercing process, which cover different regimes in view of the GN geometry and its piercing depth: ${ }^{20}$

1. $2 h_{T}<L \leq h_{H}+2 h_{T}$

$E_{i}^{\prime} /\left[2 k_{T}(1-2 \varphi) / \sin ^{2} \phi\right]= \begin{cases}-h^{2} & 0<h \leq h_{H} \\ h^{2}-4 h_{H} h+2 h_{H}^{2} & h_{H}<h \leq L / 2 \\ 3 h^{2}-4 h_{H} h-2 L h+2 h_{H}^{2}+L^{2} / 2 & L / 2<h \leq L / 2+h_{H} \\ -h^{2}+4 h_{H} h+2 L h-2 h_{H}^{2}-4 h_{H} L-L^{2} / 2 & L / 2+h_{H}<h \leq L \\ -2 h^{2}+4 h_{H} h+4 L h-2 h_{H}^{2}-4 h_{H} L-3 L^{2} / 2 & L<h \leq h_{H}+2 h_{T} \\ -4 h^{2}+8 h_{H} h+8 h_{T} h+4 L h-4 h_{H}^{2}-8 h_{T}^{2}-8 h_{H} h_{T}-4 h_{H} L-3 L^{2} / 2 & h_{H}+2 h_{T}<h \leq \mathrm{L} / 2+h_{H}+h_{T}\end{cases}$

2. $h_{H}+2 h_{T}<L \leq 2 h_{H}+2 h_{T}$

$E_{i}^{\prime} /\left[2 k_{T}(1-2 \varphi) / \sin ^{2} \phi\right]= \begin{cases}-h^{2} & 0<h \leq h_{H} \\ h^{2}-4 h_{H} h+2 h_{H}^{2} & h_{H}<h \leq L / 2 \\ h^{2}-4 h_{H} h-2 L h+2 h_{H}^{2}+L^{2} / 2 & L / 2<h \leq L / 2+h_{H} \\ -h^{2}+4 h_{H} h+2 L h-2 h_{H}^{2}-4 h_{H} L-L^{2} / 2 & L / 2+h_{H}<h \leq h_{H}+2 h_{T} \\ -3 h^{2}+8 h_{H} h+8 h_{T} h+2 L h-4 h_{H}^{2}-8 h_{T}^{2}-8 h_{H} h_{T}-4 h_{H} L-L^{2} / 2 & h_{H}+2 h_{T}<h \leq L \\ -4 h^{2}+8 h_{H} h+8 h_{T} h+4 L h-4 h_{H}^{2}-8 h_{T}^{2}-8 h_{H} h_{T}-4 h_{H} L-3 L^{2} / 2 & L<h \leq \mathrm{L} / 2+h_{H}+h_{T}\end{cases}$

3. $2 h_{H}+2 h_{T}<L \leq 4 h_{T}$

$E_{i}^{\prime} /\left[2 k_{T}(1-2 \varphi) / \sin ^{2} \phi\right]= \begin{cases}-h^{2} & 0<h \leq h_{H} \\ h^{2}-4 h_{H} h+2 h_{H}^{2} & h_{H}<h \leq L / 2 \\ 3 h^{2}-4 h_{H} h-2 L x+2 h_{H}^{2}+L^{2} / 2 & L / 2<h \leq L / 2+h_{H} \\ -h^{2}+4 h_{H} h+2 L h-2 h_{H}^{2}-4 h_{H} L-L^{2} / 2 & L / 2+h_{H}<h \leq h_{H}+2 h_{T} \\ -3 h^{2}+8 h_{H} h+8 h_{T} h+2 L h-4 h_{H}^{2}-8 h_{T}^{2}-8 h_{H} h_{T}-4 h_{H} L-L^{2} / 2 & h_{H}+2 h_{T}<h \leq 2 h_{H}+2 h_{T} \\ -2 h^{2}+4 h_{H} h+4 h_{T} h+2 L h-4 h_{T}^{2}-4 h_{H} L-L^{2} / 2 & 2 h_{H}+2 h_{T}<h \leq \mathrm{L} / 2+h_{H}+h_{T}\end{cases}$

4. $4 h_{T}<L \leq 4 h_{T}+2 h_{H}$

$E_{i}^{\prime} /\left[2 k_{T}(1-2 \varphi) \sin ^{2} \phi\right]= \begin{cases}-h^{2} & 0<h \leq h_{H} \\ h^{2}-4 h_{H} h+2 h_{H}^{2} & h_{H}<h \leq L / 2 \\ 3 h^{2}-4 h_{H} h-2 L h+2 h_{H}^{2}+L^{2} / 2 & L / 2<h \leq h_{H}+2 h_{T} \\ h^{2}+8 h_{T} h-2 L h-8 h_{T}^{2}-8 h_{H} h_{T}+L^{2} / 2 & h_{H}+2 h_{T}<h \leq L / 2+h_{H} \\ -3 h^{2}+8 h_{H} h+8 h_{T} h+2 L h-4 h_{H}^{2}-8 h_{T}^{2}-8 h_{H} h_{T}-4 h_{H} L-L^{2} / 2 & L / 2+h_{H}<h \leq 2 h_{H}+2 h_{T} \\ -2 h^{2}+4 h_{H} h+4 h_{T} h+2 L h-4 h_{T}^{2}-4 h_{H} L-L^{2} / 2 & 2 h_{H}+2 h_{T}<h \leq L / 2+h_{H}+h_{T}\end{cases}$ 
5. $4 h_{T}+2 h_{H}<L \leq 4 h_{T}+4 h_{H}$

$$
E_{i}^{\prime} /\left[2 k_{T}(1-2 \varphi) / \sin ^{2} \phi\right]= \begin{cases}-h^{2} & 0<h \leq h_{H} \\ h^{2}-4 h_{H} h+2 h_{H}^{2} & h_{H}<h \leq h_{H}+2 h_{T} \\ -h^{2}+8 h_{T} h-8 h_{T}^{2}-8 h_{H} h_{T} & h_{H}+2 h_{T}<h \leq L / 2 \\ h^{2}+8 h_{T} h-2 L h-8 h_{T}^{2}-8 h_{H} h_{T}+L^{2} / 2 & L / 2<h \leq 2 h_{H}+2 h_{T} \\ 2 h^{2}-4 h_{H} h+4 h_{T} h-2 L h+4 h_{H}^{2}-4 h_{T}^{2}+L^{2} / 2 & 2 h_{H}+2 h_{T}<h \leq L / 2+h_{H} \\ -2 h^{2}+4 h_{H} h+4 h_{T} h+2 L h-4 h_{T}^{2}-4 h_{H} L-L^{2} / 2 & L / 2+h_{H}<h \leq \mathrm{L} / 2+h_{H}+h_{T}\end{cases}
$$

6. $4 h_{T}+4 h_{H}<L$

$$
E_{i}^{\prime} /\left[2 k_{T}(1-2 \varphi) / \sin ^{2} \phi\right]= \begin{cases}-h^{2} & 0<h \leq h_{H} \\ h^{2}-4 h_{H} h+2 h_{H}^{2} & h_{H}<h \leq h_{H}+2 h_{T} \\ -h^{2}+8 h_{T} h-8 h_{T}^{2}-8 h_{H} h_{T} & h_{H}+2 h_{T}<h \leq 2 h_{H}+2 h_{T} \\ -4 h_{H} h+4 h_{T} h+4 h_{H}^{2}-4 h_{T}^{2} & 2 h_{H}+2 h_{T}<h \leq L / 2 \\ 2 h^{2}-4 h_{H} h+4 h_{T} h-2 L h+4 h_{H}^{2}-4 h_{T}^{2}+L^{2} / 2 & L / 2<h \leq L / 2+h_{H} \\ -2 h^{2}+4 h_{H} h+4 h_{T} h+2 L h-4 h_{T}^{2}-4 h_{H} L-L^{2} / 2 & L / 2+h_{H}<h \leq \mathrm{L} / 2+h_{H}+h_{T}\end{cases}
$$

For the present simulations, the oxidized degree is $\varphi=0.3$. We take typical parameter values as $h_{H}=0.5 \mathrm{~nm}, h_{T}=1.5 \mathrm{~nm}$ and $k_{T}=-7 k_{B} T / \mathrm{nm}^{2}$ in our theoretical calculations. The simulation results in Fig. $5 \mathrm{a}_{2}-\mathrm{a}_{3}$ show that the entry angle $\phi$ relative to the membrane is about $45^{\circ}$ during the piercing. Taking these detailed values into above piecewise functions, we can obtain a GN-bilayer interaction energy landscape in the space of the piercing depth and GN size, as demonstrated in Fig.7 in the main text. 


\section{Supplementary Videos}

Supplementary Video S1. Detailed transmembrane translocation process of a GN interaction with a tensionless membrane where $l_{g}=7 \mathrm{~nm}$ and $f_{R}=0.5$. Color scheme: head beads of lipids (pink); tail beads of lipids (cyan); receptors (green); the beads of the unoxidized basal plane (yellow); the beads of the oxidized edge (blue); the beads of the oxidized basal plane (red). Solvent beads are not shown for clarity.

Supplementary Video S2. Detailed capping process from an incomplete flat vesicle to a complete flat vesicle during a GN interaction with a membrane with negative tension where $l_{g}=7 \mathrm{~nm}, \sigma=-0.65 k_{B} T / r_{c}{ }^{2}$ and $f_{R}=0.5$. The color scheme is the same as that of Video S1.

Supplementary Video S3. Detailed self-rotation process during a GN interaction with a tensionless membrane where $l_{g}=7 \mathrm{~nm}$ and $f_{R}=0.5$. The color scheme is the same as that of

\section{Video S1.}

Supplementary Video S4. Detailed self-rotation process during a GN interaction with a membrane with negative tension where $l_{g}=7 \mathrm{~nm}, \sigma=-0.65 k_{B} T / r_{c}{ }^{2}$ and $f_{R}=0.5$. The color scheme is the same as that of Video S1. 


\section{References}

1. Groot, R. D.; Warren, P. B. Dissipative Particle Dynamics: Bridging the Gap between Atomistic and Mesoscopic Simulation. J. Chem. Phys. 1997, 107, 4423-4435.

2. Li, Y.; Yue, T. T.; Yang, K.; Zhang, X. Molecular Modeling of the Relationship between Nanoparticle Shape Anisotropy and Endocytosis Kinetics. Biomaterials 2012, 33, 4965-4973.

3. Vácha, R.; Martinez-Veracoechea, F. J.; Frenkel, D. Receptor-mediated Endocytosis of Nanoparticles of Various Shapes. Nano Lett. 2011, 11, 5391-5395.

4. Shi, X.; von dem Bussche, A.; Hurt, R. H.; Kane, A. B.; Gao, H. Cell Entry of One-dimensional Nanomaterials Occurs by Tip Recognition and Rotation. Nature Nanotech. 2011, 6, 714-719.

5. Guo, R.; Mao, J.; Yan, L. T. Unique Dynamical Approach of Fully Wrapping Dendrimer-like Soft Nanoparticles by Lipid Bilayer Membrane. ACS Nano 2013, 7, 10646-10653.

6. Yue, T.; Li, S.; Zhang, X.; Wang, W. The Relationship between Membrane Curvature Generation and Clustering of Anchored Proteins: a Computer Simulation Study. Soft Matter 2010, 6, 6109-6118.

7. Lerf, A.; He, H.; Forster, M.; Klinowski, J. Structure of Graphite Oxide Revisited. J. Phys. Chem. B 1998, 102, 4477-4482. 
8. Shih, C. J.; Lin, S.; Sharma, R.; Strano, M. S.; Blankschtein, D. Understanding the PH-dependent Behavior of Graphene Oxide Aqueous Solutions: Acomparative Experimental and Molecular Dynamics Simulation Study. Langmuir 2012, 28, 235-241.

9. Gomez-Navarro, C.; Meyer, J. C.; Sundaram, R. S.; Chuvilin, A.; Kurasch, S.; Burghard, M.; Kern, K.; Kaiser, U. Atomic Structure of Reduced Graphene Oxide. Nano Lett. 2010, $10,1144-1148$.

10. Mao, J.; Guo, R.; Yan, L. T. Simulation and Analysis of Cellular Internalization Pathways and Membrane Perturbation for Graphene Nanosheets. Biomaterials 2014, 35, 6069-6077.

11. Guo, R.; Mao, J.; Yan, L. T. Computer Simulation of Cell Entry of Graphene Nanosheet. Biomaterials 2013, 34, 4296-4301.

12. Min, S. H.; Lee, C.; Jang, J. Dissipative Particle Dynamics Modeling of a Graphene Nanosheet and Its Self-assembly With Surfactant Molecules. Soft Matter 2012, 8, $8735-8742$.

13. Blakslee, O. L.; Proctor, D. G.; Seldin, E. J.; Spence, G. B.; Weng, T. Elastic Constants of Compression-annealed Pyrolytic Graphite. J Appl Phys 1970, 41, 3373-82.

14. Frank, I. W.; Tanenbaum, D. M.; van der Zande, A. M.; McEuen, P. L. Mechanical Properties of Suspended Graphene Sheets. J Vac Sci Technol B 2007, 25, 2558-61.

15. Lee, C.; Wei, X.; Kysar, J. W.; Hone J. Measurement of the Elastic Properties and Intrinsic Strength of Monolayer Graphene. Science 2008, 321, 385-8. 
16. Revenga, M.; Zuniga, I.; Espanol, P. Boundary Conditions in Dissipative Particle Dynamics. Comput Phys Commun 1999, 122, 309-311.

17. Vattulainen, I.; Karttunen, M.; Besold, G.; Polson, J. M. Integration Schemes for Dissipative Particle Dynamics Simulations: from Softly Interacting Systems towards Hybrid Models. J Chem Phys 2002, 116, 3967-79.

18. Smith, K. A.; Jasnow, D.; Balazs, A. C. Designing Synthetic Vesicles that Engulf Nanoscopic Particles. J Chem Phys 2007, 127, 084703.

19. Shillcock, J. C.; Lipowsky, R. Tension-induced Fusion of Bilayer Membranes and Vesicles. Nat Mater 2005, 4, 225-8.

20. Li, Y. F.; Yuan, H. Y.; von dem Bussche, A.; Creighton, M.; Hurt, R. H.; Kane, A. B.; Gao, H. J. Graphene Microsheets Enter Cells through Spontaneous Membrane Penetration at Edge Asperities and Corner Sites. Proc. Natl Acad. Sci. USA 2013, 110, 12295-12300. 